\title{
Dynamic amplitude-phase coupling in quantum-dot lasers
}

\author{
E. Gehrig a) and O. Hess \\ Advanced Technology Institute, School of Electronics and Physical Sciences, University of Surrey, \\ Guildford, Surrey, GU2 7XH, United Kingdom
}

(Received 9 February 2005; accepted 24 April 2005; published online 13 May 2005)

\begin{abstract}
The well-known $\alpha$-factor is a convenient measure to describe the magnitude of the amplitude-phase coupling of semiconductor lasers. But is the $\alpha$-factor really a parameter? First-principle simulations of InGaAs quantum-dot lasers and amplifiers show that in spatially extended quantum-dot laser structures, the amplitude phase coupling is far from being a constant. Our computation of the $\alpha$-factor demonstrates the same large and excitation-dependent variation and scatter as corresponding to experimentally determined values. Our results therefore provide a key to the interpretation and give a measure of applicability of the $\alpha$-factor for the classification of quantum-dot lasers. (C) 2005 American Institute of Physics. [DOI: 10.1063/1.1931059]
\end{abstract}

Since the early days of laser science, the linewidth enhancement factor, or $\alpha$-factor, has been used as an important parameter for the classification of laser structures. The $\alpha$-factor is defined as the ratio of the variation of the gain and index (or real and complex parts of the complex susceptibility) with the carrier density $N$

$$
\alpha=-\frac{4 \pi}{\lambda} \frac{d n / d N}{d g / d N}=\frac{d \operatorname{Re}[\chi] / d N}{d \operatorname{Im}[\chi] / d N},
$$

where $n$ is the (induced) refractive index, $g$ is the gain, and $N$ is the carrier density. In the case of the semiconductor laser, many fundamental properties, such as linewidth, chirp, and the filamentation, are strongly influenced by the effects described by the $\alpha$-factor. In the 1980 s, first measurements revealed that the semiconductor laser linewidth was much broader than the Shawlow Townes limit. Soon after, Henry ${ }^{1}$ theoretically explained the excess linewidth with the coupling of index and gain via the carrier dynamics. Since then, the role of the $\alpha$-factor has been the very focus of many theoretical and experimental publications. ${ }^{2}$

Generally, the coupling of gain and index or amplitude and phase strongly depends on the material and cavity design of the semiconductor medium. Semiconductor quantum dots (QDs) have discrete level energies and a rather symmetric gain and induced index dispersion. QD lasers (QDLs) thus should, in principle, have a small $\alpha$-factor. Indeed, values near 1.0 have been found for InGaAs/GaAs QDLs in experiments. ${ }^{3}$ Furthermore, it has been demonstrated that the $\alpha$-factor in QDLs is much lower and shows less dispersion than in quantum-well lasers of identical material and geometry. ${ }^{4}$ However, the measured values of $\alpha$ strongly vary, even in the same type of QDL from device to device. ${ }^{5}$ One may thus immediately ask: Is $\alpha$ really a factor? Is it really is a good quantity for the description and classification of the optical properties of QD semiconductor lasers taking into account the characteristic spatial disorder of QD ensembles in temporal variation and spatial-temporal average?

To shed light on these questions, we study the amplitude-phase coupling dynamics in QDL and calculate the $\alpha$-factor based on first principles. The theoretical description is based on spatially resolved QD Maxwell-Bloch equa-

$\overline{{ }^{a} \text { Electronic mail: e.gehrig@surrey.ac.uk }}$ tions that describe the spatiotemporal light field and inter-/ intralevel carrier dynamics in each QD of a typical QD ensemble. ${ }^{6}$ In particular, this includes spontaneous luminescence, counterpropagation of amplified spontaneous emission, and induced recombination as well as carrier diffusion in the wetting layer of the laser. Intradot scattering via emission and absorption of phonons, as well as the scattering with the carriers and phonons of the surrounding wetting layer are dynamically included on a mesoscopic level. Spatial fluctuations in size and energy levels of the QDs and irregularities in the spatial distribution of the QDs in the active layer are simulated via statistical methods. Although, the explicit consideration of the full dipole dynamics does not require an artificial inclusion of the $\alpha$ parameter, we can in our equations extract the value of this parameter via the dynamically calculated real and imaginary part of the polarization that are via the light fields directly correlated to the susceptibility. In the following, we will address the following questions: (i) How does the characteristic spatial disorder of QD ensembles and the light-field dynamics affect $\alpha$ ? (ii) How does the ultrafast dynamcis feed back onto $\alpha$ ?

For a QDL of $1 \mathrm{~mm}$ length and $50 \mu \mathrm{m}$ stripe width Fig. 1 shows the dynamics of the (spatially averaged) amplitude phase coupling after the startup of the laser (i.e., switching

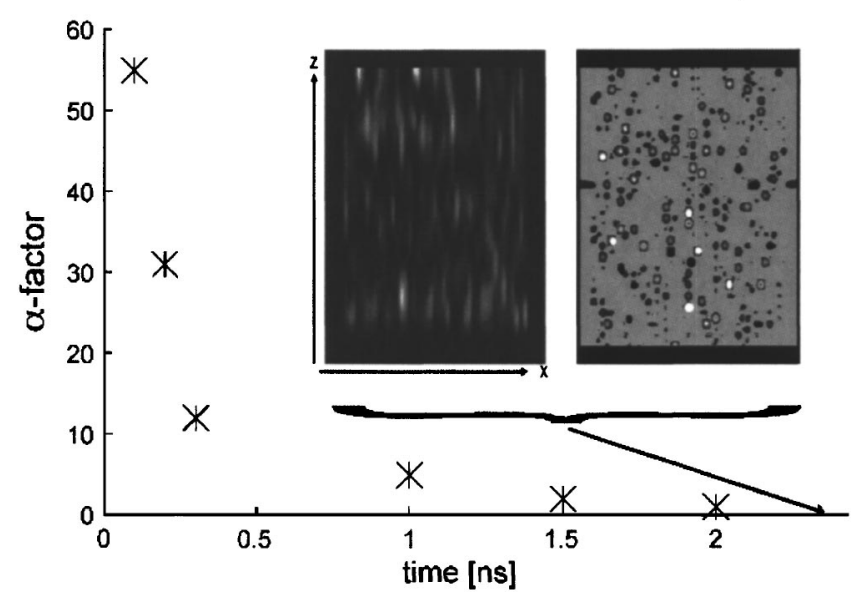

FIG. 1. Dynamics of $\alpha$ in a QDL $(L=1 \mathrm{~mm}, w=50 \mu \mathrm{m})$ : Spatial average dependent of time immediately after the switching on the current. Insert: Snapshots of the intensity (left) and $\alpha$ (right) at $2.5 \mathrm{~ns}$. 

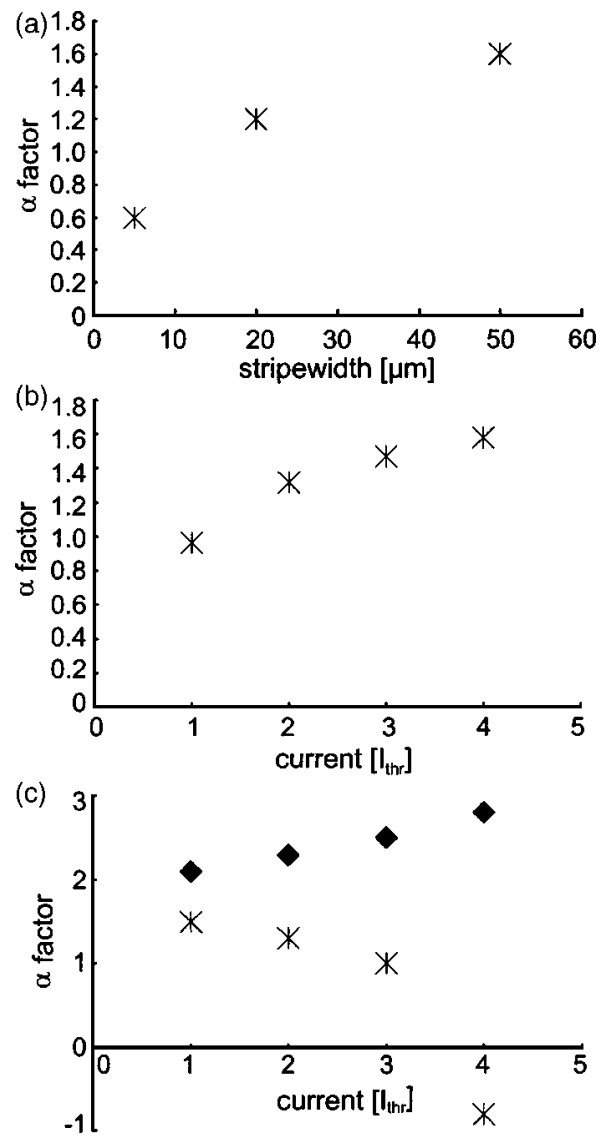

FIG. 2. Dependence of alpha on stripe width (a) and on injection current [(b) and (c)] for a stripe width of $6 \mu \mathrm{m}$ (b) and $50 \mu \mathrm{m}$ (c). Stars and diamonds in (c) represent homogeneous and inhomogeneous dots, respectively.

the current from zero to its final value). The insert visualizes a snapshot (taken after $2.5 \mathrm{~ns}$ ) of the spatial distribution of the intensity (left) and the $\alpha$-factor (right). The spatial average of $\alpha$ shows a relaxation behavior that orginates from the out-of-equilibrium dynamics of light fields and carriers within the first few nanoseconds. The snapshot shows an image of dynamic $\mu \mathrm{m}$-sized light patterns frozen in time. The formation of this pattern originates from the dynamic light-matter coupling characterized by light diffraction, selffocusing, and complex carrier scattering. At the same time, the spatially varying carrier and intradot dipole dynamics leads to the highly complex dynamics and the strong spatial dependence of the $\alpha$-factor. Indeed, typical values for $\alpha$ may, in a given laser structure, spatially and temporally vary from values between -3 to 3 or even show singularities while the average with respect to space and time may be considerably lower. Although $\alpha$ may be comparatively small in each individual dot at a particular moment in time the complex amplitude and phase dynamics resulting from the characteristic spatially varying material properties (e.g., energy levels and dipole matrix elements in combination with the dynamic coupling to the counterpropagating light fields) lead to strong spatial and temporal changes in $\alpha$. The $\alpha$ "factor" consequently is far from being constant: Fig. 2(a) shows the dependence of $\alpha$ (temporally and spatially averaged) on the width of the laser while Figs. 2(b) and 2(c) demonstrate the dependence of $\alpha$ on carrier injection for a narrow-stripe laser (stripe width of $6 \mu \mathrm{m}$ ) and a broad-stripe laser (transverse stripe width $50 \mu \mathrm{m}$ ), respectively. With increasing width of the QDL, the transverse dimension effectively becomes more and more important: Physical processes, such as carrier diffusion, light diffraction, and dynamic phase changes, result in a notable deterioration of the beam quality ${ }^{7}$ and an increase in $\alpha$. In the narrow-stripe QDL (width of $6 \mu \mathrm{m}$ ) $\alpha$ increases slightly with increasing carrier density. There, the increase in carrier injection induces a strong intradot carrier dynamics characterized by carrier capture and carrier-phonon interactions coupling the various energy levels within the dot. As a consequence, the carrier-induced gain and index dispersion show complex dynamics leading to an increase in $\alpha$. In the QDL with the large stripe width, we find an additional increase in complexity: For an ideal laser with homogeneous material properties (i.e., identical dot size, matrix elements, and uniform dot distribution), the amplitude phase coupling may even decrease with increasing stripe with. This effect can be attributed to strong Coulomb interactions that may shift the characteristic gain and index distributions as well as the resulting $\alpha$ parameter. ${ }^{8}$ In the spatially inhomogeneous dot ensemble (in the example: $18 \mathrm{meV}$ ), the amplitude phase coupling rises with increasing carrier injection due to the increase in spatiotemporal carrier, gain, and index dynamics.

Figure 3 shows a snapshot of the intensity (a) and the $\alpha$ factor (b) in the active area of a QD laser amplifier (width $6 \mu \mathrm{m}$, length $1 \mathrm{~mm}$ ) into which a resonant picosecond light pulse has been injected. The optical injection leads to a dynamic excitation and relaxation within the charge carrier system. This is directly reflected in the rise and decrease of $\alpha$ [Fig. 3(b)]. Under these particular conditions, $\alpha$ represents the material response to the optical excitation and more or less mirrors the pulse shape. We would like to note that the specific shape and (spatial as well as temporal) offset of $\alpha$

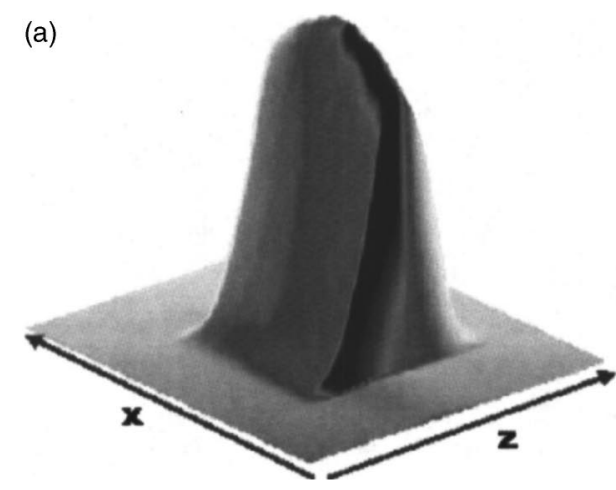

(b)

FIG. 3. Snapshots of intensity (a) and alpha (b) during the propagation of a light pulse. 

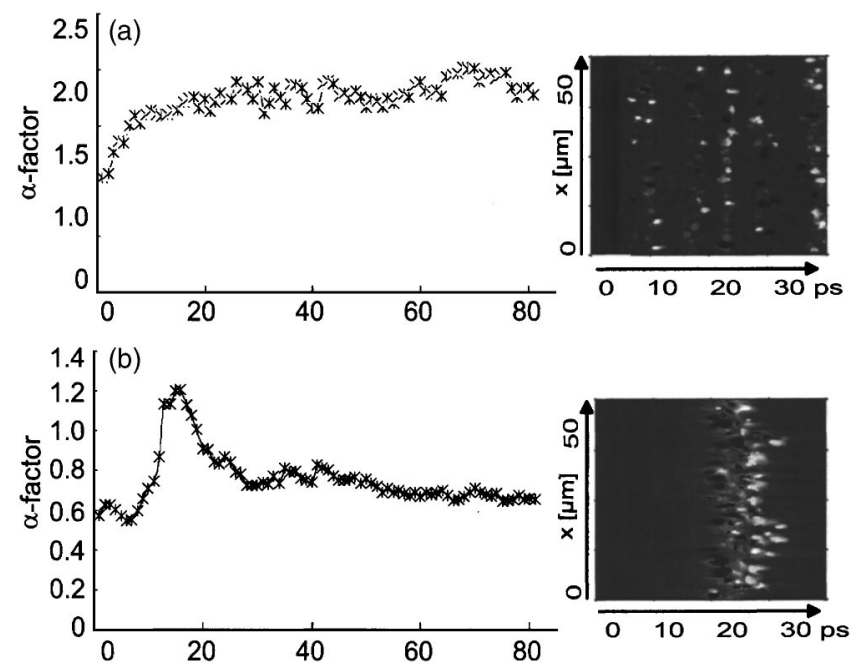

FIG. 4. Dynamics of alpha during the propagation of a light pulse.

strongly depends on carrier inversion and the degree of saturation. In strongly saturated amplifiers the spatial distribution of $\alpha$ may be significantly distorted while the propagating pulse is significantly reshaped. Furthermore, in laser amplifiers with large stripe widths $\alpha$ may show a strong transverse dependence. Characteristic of the ultrashort regime, Fig. 4 shows the spatially averaged $\alpha$ in dependence on time during the passage of a 150 fs light pulse in a QDL amplifier (stripe width $50 \mu \mathrm{m}$ ) with (a) a spatially inhomogeneous and (b) a homogeneous ensemble of QD. The figures on the right visualize the respective spatially resolved dynamics of $\alpha$ at the output facet. In the spatially inhomogeneous dot system, the light pulse induces a highly nonequilibrium situation in the charge carrier system. It is the dynamic interplay of counterpropagating light fields and spatially dependent dipole dynamics that leads to the dynamic spatial phase pattern in the active area. As a consequence, the nonequilibrium dynamics induced by the light pulse may lead to a long-lived excitation [Fig. 4(b)]. In the homogeneous dot ensemble [Fig. 4(a)], the overall relaxation of induced gain and index is faster leading to a characteristic rise and descrease in $\alpha$.

In conclusion, we have presented numerical results on the spatiotemporal dynamics of the $\alpha$-factor in QDLs. The explicit inclusion of the spatially varying carrier distributions and the complex intradot dipole dynamics in the QD Maxwell-Bloch equations provides a fundamental description of the underlying physical processes and guarantees a realistic modeling of the laser-internal amplitude phase coupling. In particular, the simulations allow the systematic variation of the individual parameters and properties with respect to their influence on $\alpha$. Our spatiotemporally resolved calculations not only predict the average $\alpha$ factor but also reveal the correlation between spatiotemporal light-field and carrier dynamics and the complex dynamics of the amplitude phase coupling. The results obtained on this basis clearly demonstrate that-in spite of the small spatiotemporal average-the $\alpha$ factor in QDLs shows a complex spatial and temporal dynamics. Although a measured $\alpha$ parameter (or the average of a calculation) may be appropriate for a first evaluation of the optical properties of a QDL device, one has to be aware that the laser-internal amplitude phase coupling is a strongly varying physical quantity affected by complex light-matter interactions and carrier dynamics.

Support by the European project DOTCOM (IST-200028713) is gratefully acknowledged.

${ }^{1}$ C. H. Henry, IEEE J. Quantum Electron. 18, 259 (1982).

${ }^{2}$ M. Osinski and J. Buus, IEEE J. Quantum Electron. 23, 9 (1987).

${ }^{3}$ S. Ghosh, S. Pradhan, and P. Bhattacharya, Appl. Phys. Lett. 81, 3055 (2002).

${ }^{4}$ A. A. Ukhanov, A. Stintz, P. G. Eliseev, and K. J. Malloy, Appl. Phys. Lett. 84, 1058 (2004).

${ }^{5}$ S. Schneider, P. Borri, W. Langbein, U. Woggon, R. L. Sellin, D. Ouyang, and D. Bimberg, IEEE J. Quantum Electron. 40, 1423 (2004).

${ }^{6}$ E. Gehrig and O. Hess, Phys. Rev. A 65, 033804 (2002).

${ }^{7}$ E. Gehrig, O. Hess, C. Ribbat, R. L. Sellin, and D. Bimberg, Appl. Phys. Lett. 84, 1650 (2004).

${ }^{8}$ P. M. Smowton, E. J. Pearce, H. C. Scheider, W. W. Chow, and M. Hopkinson, Appl. Phys. Lett. 81, 3251 (2002). 